\title{
Phenolic Compounds and Antioxidant Activity of Rice
}

\author{
Melissa Walter ${ }^{*}$ and Enio Marchesan ${ }^{2}$ \\ ${ }^{1}$ Instituto Federal de Educação, Ciência e Tecnologia Farroupilha; Campus Santa Rosa; 98900-000; Santa Rosa - \\ $R S$ - Brasil. ${ }^{2}$ Departamento de Fitotecnia; Centro de Ciências Rurais; Universidade Federal de Santa Maria; Santa \\ Maria - RS - Brasil
}

\begin{abstract}
The aim of this work was to study the phenolic compounds identified in rice, their antioxidant activity and their potential beneficial effects on health. In vitro and in vivo studies evaluating the rice grains with different pericarp colour (light brown, red and black) showed potential beneficial effects on health related to the polyphenol content of the grain, such as reduction of oxidative stress, aid in the prevention of cancer, cardiovascular diseases and complications of diabetes, among others.
\end{abstract}

Key words: rice, red rice, black rice, polyphenols, antioxidant, biological effect

\section{INTRODUCTION}

Several studies have demonstrated the importance of diet in the control of chronic diseases, such as cancer and cardiovascular problems (Birt et al., 2001; Kris-Etherton et al., 2002; Stanner et al., 2003; Houston, 2005). The consumption of fruits, legumes, vegetables and whole grains results a reduced risk of developing these diseases. This could be attributed to the presence of natural antioxidants in these foods, such as ascorbic acid, tocopherols, carotenoids and phenolic compounds (polyphenols) (Meléndez-Martínez et al., 2004; Choi et al., 2007), besides other bioactive compounds.

Among the compounds with antioxidant activity, polyphenols are important. These are found in a great variety of foods, such as apples, mulberries, cherries, grapes, raspberries, citric fruits, onions, spinach, peppers, oat, wheat, black tea, wine and chocolate, among others (Holden et al., 2005;
Dimitrios, 2006). These compounds have demonstrated higher in vitro antioxidant capacity than other antioxidants, such as ascorbic acid and $\alpha$-tocopherol (Pulido et al., 2000), emphasising the importance of polyphenols as antioxidants in the diet.

Although polyphenols are found in several foods, variation is observed in the concentration and type of these compounds due to genetic and environmental factors and processing conditions (Kris-Etherton et al., 2002). Hence, the concentration of phenolic compounds varies greatly among the diets, depending on the type and quantity of food consumed.

Rice, being one of the most produced and consumed cereals in the world, has an important role in the relation between the diet and health. Several compounds with antioxidant activity have been identified in rice, including phenolic compounds, tocopherols, tocotrienols and $\gamma$ oryzanol (Iqbal et al., 2005). The phenolic

*Author for correspondence: melmelissaw@ hotmail.com 
compounds are mainly associated with the pericarp in rice, hence, the milling process reduces the concentration of these compounds in the grain. Besides, grains with darker pericarp colour, such as red and black rice, contain higher amounts of polyphenols (Tian et al., 2004; Zhou et al., 2004).

The concentration of total phenolics in the grain has been positively associated with the antioxidant activity (Itani et al., 2002; Goffman and Bergman, 2004; Zhang et al., 2006), with potential beneficial effects on health, such as reduction of oxidative stress (Ling et al., 2001; Hu et al., 2003), aid in the prevention of cancer (Hudson et al., 2000; Hu et al., 2003; Hyun and Chung, 2004; Chen et al., 2006), in the control of blood lipids and related diseases, which may help in the prevention of cardiovascular problems (Ling et al., 2001), and in the prevention of the complications of diabetes (Morimitsu et al., 2002; Yawadio et al., 2007).

The aim of this article was to examine the phenolic compounds present in rice, their antioxidant activity and their potential beneficial effects on health.

\section{PHENOLIC COMPOUNDS}

Phenolic compounds are secondary metabolites of plants, with different activities such as protection against pathogens and predators, mechanical support, attraction of pollinating animals, and protection against ultraviolet radiation (Bravo, 1998; Parr and Bolwell, 2000). These compounds constitute a chemically heterogeneous group, containing a phenol group (a functional hydroxyl group in an aromatic ring) in its basic structure. They differ structurally from simple molecules, such as phenolic acids, to highly polymerised compounds, such as tannins, comprising different classes. However, the main phenolics in the diet are the phenolic acids, flavonoids and tannins (King and Young, 1999).

Phenolic acids consist of two subgroups, the benzoic acid and cinnamic acid derivatives (Balasundram et al., 2006). With a $\mathrm{C}_{6}-\mathrm{C}_{1}$ structure (an aromatic ring linked to a carbon atom), the benzoic acid derivatives include $\rho$-hidroxybenzoic, protocatechuic, vanillic, gallic and syringic acids. The cinnamic acid derivatives, with a $\mathrm{C}_{6}-\mathrm{C}_{3}$ structure (an aromatic ring linked to a three-carbon chain), include caffeic, ferulic, $\rho$-coumaric and sinapic acids (Simões et al., 2001). These acids may be in the free form, in the form of soluble conjugates or in the insoluble bound form (Adom and Liu, 2002; Zhou et al., 2004). Bound phenolic acids are typically involved in the structure of the cell wall, doing the cross-linking of lignin components, with effects on the growth of the cell wall, its mechanical properties and degradability (Zhou et al., 2004).

Flavonoids, the major class of phenolic compounds in plants, are formed by 15 carbons, organised in two aromatic rings linked by a threecarbon chain (structure $\mathrm{C}_{6}-\mathrm{C}_{3}-\mathrm{C}_{6}$ ) (Rossi and Kasum, 2002). They can be divided into different classes, being the anthocyanidins the most common, responsible for the majority of the red, pink, purple and blue colours in the plants, acting as attraction for the animals for pollination and seed dispersal. Generally, the anthocyanidins are bound to glycosides, which are called anthocyanins (Kong et al., 2003).

Tannins form another group of phenolic polymers with defence properties for the plant and can be divided into condensed and hydrolysable tannins. Condensed tannins are formed by the polymerisation of flavonoid units. These compounds are frequently hydrolysed to anthocyanidins, hence, they are also called proanthocyanidins. The hydrolysable tannins are heterogeneous polymers containing phenolic acids, especially gallic acid, and simple sugars (SantosBuelga and Scalbert, 2000; Balasundram et al., 2006).

\section{PHENOLIC COMPOUNDS AND ANTIOXIDANT ACTIVITY}

Besides their functions in vegetables, studies have demonstrated the beneficial effects of phenolic compounds from different sources in human health due to their antioxidant activity. Cells are continuously exposed to oxidants from endogenous and exogenous sources and the production of free radicals is a part of the metabolism. The organism also has antioxidant compounds from endogenous and exogenous sources participating in the maintenance of the balance between oxidants and antioxidants (Benzie and Szeto, 1999; Fogliano et al., 1999; Heim et al., 2002). The disequilibrium in this balance due to the alteration in the concentration of these compounds is called oxidative stress, which results 
in damage to the cells and tissues in several ways: damaging the biomolecules and cell components, activating specific signalling paths, originating toxic products, changing gene expression and enzyme activity, and interrupting normal mechanisms of cell repair. For these reasons, oxidative stress has been related to several chronic diseases, including cardiovascular diseases, aging, diabetes and cancer (Stanner et al., 2003).

Phenolic compounds may exert their antioxidant activity in different ways. They may directly scavenge some reactive species, including hydroxyl, peroxyl and superoxide radicals, acting as chain breaking antioxidants. They may suppress lipid peroxidation recycling other antioxidants, such as $\alpha$-tocopherol. Some phenolic compounds may bind pro-oxidant metals, such as iron and copper, preventing the formation of free radicals from these pro-oxidants while simultaneously maintaining their capacity to scavenge free radicals (Moran et al., 1997; Kris-Etherton et al., 2003; Halliwell, 2007). Besides, the effects of some phenolics are related to the increase in the activity of antioxidant enzymes (Chiang et al., 2006) and induction of the synthesis of antioxidant proteins (Chung et al., 2006).

\section{PHENOLIC COMPOUNDS IN RICE}

Several phenolic compounds have already been identified in rice. While grains with light brown pericarp color present mainly low molecular weight phenolics (approximately 85\%), in those with red and black pericarp color prevail the compounds with higher molecular weight (Goffman and Bergman, 2004). The main phenolics in rice grains with light brown pericarp color are the phenolic acids, mainly ferulic and $\rho$ coumaric acids (Tian et al., 2004; Zhou et al., 2004). Other compounds identified include sinapic acid, protocatechuic acid (Hudson et al., 2000; Tian et al., 2005), chlorogenic acid, hidroxybenzoic acid (Tian et al., 2005), vanillic acid, syringic acid (Zhou et al., 2004; Tian et al., 2005), caffeic acid (Hudson et al., 2000; Zhou et al., 2004; Tian et al., 2005) and gallic acid (Zhou et al., 2004), tricin (flavone) (Hudson et al., 2000) and the esters 6'-O-(E)-feruloylsucrose and 6'-O(E)-sinapoylsucrose (Tian et al., 2004; Tian et al., 2005).
In rice grains with red and black pericarp, the main phenolics are the anthocyanins cyanidin-3-O- $\beta$-Dglucoside and peonidin-3-O- $\beta$-D-glucoside (Oki et al., 2002; Hu et al., 2003; Chen et al., 2006; Zhang et al., 2006; Yawadio et al., 2007). Other compounds identified include the anthocyanidins cyanidin and malvidin (Hyun and Chung, 2004; Zhang et al., 2006), the anthocyanins pelargonidin3,5-diglucoside and cyanidin-3,5-diglucoside (Zhang et al., 2006) and phenolic acids, like ferulic, caffeic and protocatechuic acids (Morimitsu et al., 2002).

According to Yawadio et al. (2007), the main characteristic that determined the type of phenolic compounds in the grain is pericarp colour, as no differences were observed among the anthocyanins when evaluating the grains with black pericarp from the subspecies indica and japonica. Pericarp colour is also related to the concentration of phenolics in the grain and usually the concentration is higher in the grains with red and black pericarp. Goffman and Bergman (2004), evaluating different genotypes, obtained total phenolic content between 1.90 and $50.32 \mathrm{mg}$ GAE (gallic acid equivalent) $\mathrm{g}^{-1}$ bran, and between 0.25

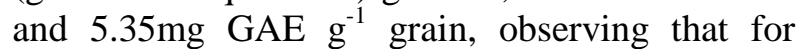
those genotypes with light brown pericarp colour these values were the lowest. Besides the difference in the content of total phenolics related to the colour of the grains, variation was also observed in the content of total phenolics among the genotypes with the same pericarp colour.

Besides the genetic characteristic, other factors may influence the concentration of phenolics. Although no influence of the growing season was observed in the content of these compounds (Goffman and Bergman, 2004), different studies have demonstrated the effect of grain processing, including polishing and germination. Polishing significantly reduces the concentration of phenolics since these compounds are localized mainly in the external layers of the grain. The bran contains between 70 and $90 \%$ of the phenolic acids in light brown pericarp rice grains (Zhou et al., 2004), and approximately $85 \%$ of the anthocyanins in the rice grains with black pericarp (Hu et al., 2003), with little variation depending on the cultivar and the compounds considered. According to Goffman and Bergman (2004), this high correlation between the content of phenolics in the grain and in the bran showed that it was 
possible to select for higher or lower phenolics content through the analysis of the grain without polishing, reducing the time of sample preparation. The germination process also affects the phenolic compounds in the grain. Tian et al. (2005) observed a reduction of approximately $70 \%$ in the concentration of feruloylsucrose and sinapoylsucrose, with an increase in the content of ferulic and sinapic acids in the grains of rice with light brown pericarp during the germination. This reduction was probably caused by the hydrolysis, indicating that the germination caused the metabolism of phenolic compounds.

Some studies have also shown that the distribution of phenolic compounds changed during the storage. Zhou et al. (2004) observed a reduction in the content of bound phenolic acids in brown and white rice during the storage; this reduction was higher at $37^{\circ} \mathrm{C}$ than at $4^{\circ} \mathrm{C}$. In contrast, the concentration of free phenolic acids in white rice significantly increased during the storage, probably as a result of the enzymatic or nonenzymatic release of the bound phenolic acids.

\section{ANTIOXIDANT ACTIVITY AND BIOLOGICAL EFFECT}

The concentration of total phenolics in the rice grains has been positively correlated with the antioxidant activity (Itani et al., 2002; Goffman and Bergman, 2004; Zhang et al., 2006). In red pericarp grains, a high correlation was observed between this activity and the content of proanthocyanidins, but in the case of black pericarp grains the correlation depended on the content of anthocyanins (Oki et al., 2002). These results suggested that the phenolic compounds were among the main responsible ones for the antioxidant activity of rice grains (Goffman and Bergman, 2004).

Usually, grains with red and black pericarp presented higher antioxidant activity than those with light brown pericarp colour (Nam et al., 2005). Goffman and Bergman (2004), evaluating the genotypes with different pericarp colour, observed the values of antioxidant activity between 10.0 and $13.1 \mu \mathrm{M}$ TE (Trolox equivalent) $\mathrm{g}^{-1}$ bran for the grains with light brown pericarp, between 119.9 and $312.3 \mu \mathrm{M} \mathrm{TE} \mathrm{g}^{-1}$ bran for red pericarp grains and between 56.3 e $345.3 \mu \mathrm{M}^{\mathrm{TE}} \mathrm{g}^{-}$ ${ }^{1}$ bran for black pericarp grains. These results also demonstrated that, besides the variation in the antioxidant activity among grains with different pericarp colour, variation in a group of grains with the same pericarp colour was also observed.

Besides the difference in the total antioxidant activity, differences have also been observed among the genotypes in the ability to scavenge the reactive oxygen species. Nam et al. (2005) reported reduction in the concentration of superoxide anions by competitively inhibiting xanthine oxidase (enzyme that induces the formation of reactive oxygen species in the cells), and scavenged hydroxyl radicals through direct mechanism. On the other hand, another genotype scavenged superoxide anions without affecting the activity of xanthine oxidase, and reduced hydroxyl radicals through binding ferrous ion. This showed that the antioxidant compounds present in these grains might act in different ways to reduce the oxidative stress in the organism and, with that, help in the prevention of several diseases.

The reduction of the oxidative stress by polyphenols in rice grains was observed in in vitro and ex vivo studies, indicated by the reduction in the production of nitric oxide (Hu et al., 2003) and in the concentration of reactive oxygen species (Hu et al., 2003; Chiang et al., 2006). Simultaneously to the reduction in the indicators of oxidation, an increase in the antioxidant capacity was observed, including higher total antioxidant capacity and increased activity of antioxidant enzymes, such as superoxide dismutase and catalase (Chiang et al., 2006). Studies with cell cultures have shown that phenolic compounds in the rice may also be associated with antimutagenic, anticarcinogenic and antimetastasis activities, due to their ability to directly protect the DNA against the damage and affect cell proliferation (Hu et al., 2003; Chen et al., 2006). Using the extracts obtained from the rice grains with light brown pericarp colour, a reduction in the number of viable cells and colony formation of breast and colon cancer cells was observed (Hudson et al., 2000). Evaluating separately some phenolic compounds present in the rice bran, effects were observed on different types of cancer cells. Caffeic acid, ferulic acid and tricin reduced the number of cells, cell viability and clonogenicity (Hudson et al., 2000). The anthocyanins cyanidin and malvidin presented cytotoxicity in a dose-dependent way, with $\mathrm{IC}_{50}$ values (concentration that inhibited the growth by 
$50 \%$ ) for human leukaemia cells as 60 and $40 \mu \mathrm{g}$ $\mathrm{ml}^{-1}$, respectively. This activity was attributed to the effect of these compounds on cell cycle, interrupting it at $\mathrm{G}_{2} / \mathrm{M}$ phase and inducing the apoptosis. However, no cytotoxic activity was observed when these compounds were evaluated in the form of glucosides, suggesting that this activity was due to the aglycone part of the molecule (Hyun and Chung, 2004). Peonidin-3glucoside and cyanidin-3-glucoside showed inhibition of cell invasion and motility of human hepatocellular carcinoma cells, without apparent toxicity (Chen et al., 2006).

The extracts obtained from the rice with red and black pericarp may also have positive effects in the prevention of the complications of diabetes. Morimitsu et al. (2002) observed an inhibitory effect on the lens opacity in lens organ culture obtained from the rats; this might help in the prevention of cataract in diabetics. According to them, this effect might be related to the inhibition of the enzyme aldose reductase. Some compounds were isolated from the black rice and tested in vitro, including cyanidin, peonidin, ferulic acid and $\alpha$-tocopherol, which demonstrated an inhibitory effect on this enzyme with a dosedependent effect (Yawadio et al., 2007).

Although in vitro studies can give some information about the antioxidant activity and possible biological effects of rice phenolics, the relevance of this information to antioxidant effectiveness in the organism is very limited without data on their bioavailability and metabolism (Collins, 2005). The bioavailability differs greatly from one polyphenol to another due to several factors, such as food matrix, concentration of the polyphenol in the food, background diet and interindividual variations, affecting the concentration of active metabolites in the organism (Manach et al., 2005; Zhao and Moghadasian, 2008). However, few studies have been developed to evaluate the antioxidant properties of rice phenolics in vivo, and most of them used animal models instead of humans. Studies with animals have shown beneficial effects of the consumption of the colored fraction (pericarp) of rice grains on the control of blood lipids and related diseases, helping in the prevention of cardiovascular problems. In these studies, with apolipoprotein (apo)E-deficient mice (Xia et al., 2003) and hypercholesterolemic rabbits (Ling et al., 2001), the supplementation of the diet with the pericarp of the rice reduced the occurrence of atherosclerotic plaques. This effect was related to different mechanisms, including the increase in the antioxidant capacity of the organism (Ling et al., 2001), reduction in the total cholesterol concentration in the blood (Xia et al., 2003), reduction in the ratio between LDL and HDL cholesterol (Ling et al., 2001; Xia et al., 2003), reduction in the accumulation of cholesterol in the aorta tissue and reduction in the oxidation of LDL cholesterol (Xia et al., 2003).

A study with humans was developed by Wang et al. (2007) aiming at evaluating the effect of diet supplementation of black rice fraction in the patients with coronary heart disease. The patients consuming the black rice fraction showed an increase in the plasma antioxidant status and reduction in inflammation. This could benefit the patients with coronary heart disease. According to the authors, the higher amount of anthocyanins contained in the black rice compared to white rice might be the major component responsible for the cardioprotective effects observed. The absorption of cyanidin-3-O- $\beta$-D-glucoside (the dominant anthocyanin in black rice) was also observed, which appeared in the plasma and reached maximum level $(21.5 \pm 4.48 \mathrm{ng} / \mathrm{ml})$ at $1.5 \mathrm{~h}$, but disappeared quickly after $4 \mathrm{~h}$.

\section{CONCLUSIONS}

Phenolic compounds, due to their antioxidant activity, present potential beneficial health effects. The same variability observed in the concentration of these compounds in the foods is also observed in the diets, and the inclusion in the diet of foods rich in these compounds may change the balance between the oxidants and antioxidants in the organism, which may help in the maintenance of health. Rice possesses different compounds with antioxidant activity, including polyphenols, but variations are observed in the concentration of these compounds in the grains, mainly due to genotype, pericarp colour and processing.

In vitro and in vivo studies have shown potential beneficial effects of rice phenolics on the health. However, the majority of these studies have evaluated the effect of fractions rich in phenolics, and not the grain itself, as it is usually consumed. Hence, more studies should be done evaluating the effect of the consumption of the grain, helping to 
understand if the consumption of rice in the diet can have beneficial effects on the health. Although rice is not among the foods with higher concentrations of phenolic compounds, it may be an important source of these compounds due to its wide utilization in feeding, justifying the studies in this area, especially in in vivo studies, to evaluate the bioavailability and metabolism of rice polyphenols and their effect on the organism.

\section{ACKNOWLEDGMENTS}

The authors acknowledge the financial support granted by "Conselho Nacional de Desenvolvimento Científico e Tecnológico (CNPq)".

\section{REFERENCES}

Adom, K. K. and Liu, R. H. (2002), Antioxidant activity of grains. J Agric Food Chem, 50, 61826187.

Balasundram, N.; Sundram, K. and Samman, S. (2006), Phenolic compounds in plant and agri-industrial byproducts: antioxidant activity, occurrence, and potential uses. Food Chem, 99, 191-203.

Benzie, I. F. F. and Szeto, Y. T. (1999), Total antioxidant capacity of teas by the ferric reducing/antioxidant power assay. J Agric Food Chem, 47, 633-636.

Birt, D. F.; Hendrich, S. and Wang, W. (2001), Dietary agents in cancer prevention: flavonoids and isoflavonoids. Pharmacol Therapeut, 90, 157-177.

Bravo, L. (1998), Polyphenols: chemistry, dietary sources, metabolism, and nutritional significance. Nutr Rev, 56, 317-333.

Chen, P.; Kuo, W.; Chiang, C.; Chiou, H.; Hsieh, Y. and Chu, S. (2006), Black rice anthocyanins inhibit cancer cells invasion via repressions of MMPs and uPA expression. Chem-Biol Interact, 163, 218-229.

Chiang, A.; Wu, H.; Yeh, H.; Chu, C.; Lin, H. and Lee, W. (2006), Antioxidant effects of black rice extract through the induction of superoxide dismutase and catalase activities. Lipids, 41, 797-803.

Choi, Y.; Jeong, H. S. and Lee, J. (2007), Antioxidant activity of methanolic extracts from some grains consumed in Korea. Food Chem, 103, 130-138.

Chung, M. J.; Walker, P. A. and Hogstrand, C. (2006), Dietary phenolic antioxidants, caffeic acid and Trolox, protect rainbow trout gill cells from nitric oxide-induced apoptosis. Aquat Toxicol, 80, 321-328.
Collins, A. R. (2005), Assays for oxidative stress and antioxidant status: applications to research into the biological effectiveness of polyphenols. Am J Clin Nutr, 81, 261S-267S.

Dimitrios, B. (2006), Sources of natural phenolic antioxidants. Trends Food Sci Tech, 17, 505-512.

Fogliano, V.; Verde, V.; Randazzo, G. and Ritieni, A. (1999), Method for measuring antioxidant activity and its application to monitoring the antioxidant capacity of wines. J Agric Food Chem, 47, 10351040 .

Goffman, F. D. and Bergman, C. J. (2004), Rice kernel phenolic content and its relationship with antiradical efficiency. J Sci Food Agr, 84, 1235-1240.

Halliwell, B. (2007), Dietary polyphenols: good, bad, or indifferent for your health? Cardiovasc Res, 73, 341347.

Heim, K. E.; Tagliaferro, A. R. and Bobilya, D. J. (2002), Flavonoid antioxidants: chemistry, metabolism and structure-activity relationships. $J$ Nutr Biochem, 13, 527-584.

Holden, J. M.; Bhagwat, S. A.; Haytowitz, D. B.; Gebhardt, S. E.; Dwyen, J. T.; Peterson, J.; Beecher, G. R.; Eldridge, A. L. and Balentine, D. (2005), Development of a database of critically evaluated flavonoids data: application of USDA's data quality evaluation system. J Food Compos Anal, 18, 829844.

Houston, M. C. (2005), Nutraceuticals, vitamins, antioxidants, and minerals in the prevention and treatment of hypertension. Prog Cardiovasc Dis, 47, 396-449.

Hu, C.; Zawistowski, J.; Ling, W. and Kitts, D. D. (2003), Black rice (Oryza sativa L. indica) pigmented fraction suppresses both reactive oxygen species and nitric oxide in chemical and biological model systems. J Agric Food Chem, 51, 5271-5277.

Hudson, E. A.; Dinh, P. A.; Kokubun, T.; Simmonds, M. S. J. and Gescher, A. (2000), Characterization of potentially chemopreventive phenols in extracts of brown rice that inhibit the growth of human breast and colon cancer cells. Cancer Epidem Biomar, 9, 1163-1170.

Hyun, J. W. and Chung, H. S. (2004), Cyanidin and malvidin from Oryza sativa cv. Heugjinjubyeo mediate cytotoxicity against human monocytic leukemia cells by arrest of $\mathrm{G}_{2} / \mathrm{M}$ phase and induction of apoptosis. J Agric Food Chem, 52, 2213-2217.

Iqbal, S.; Bhanger, M. I. and Anwar, F. (2005), Antioxidant properties and components of some commercially available varieties of rice bran in Pakistan. Food Chem, 93, 265-272.

Itani, T.; Tatemoto, H.; Okamoto, M.; Fujii, K. and Muto, N. (2002), A comparative study on antioxidative activity and polyphenol content of colored kernel rice. J Jpn Soc Food Sci, 49, 540-543. 
King, A. and Young, G. (1999), Characteristics and occurrence of phenolic phytochemical. J Am Diet Assoc, 99, 213-218.

Kong, J.; Chia, L.; Goh, N.; Chia, T. and Brouillard, R. (2003), Analysis and biological activities of anthocyanins. Phytochemistry, 64, 923-933.

Kris-Etherton, P. M.; Hecker, K. D.; Bonanome, A.; Coval, S. M.; Binkoski, A. E.; Hilpert, K. F.; Griel, A. E. and Etherton, T. D. (2002), Bioactive compounds in foods: their role in the prevention of cardiovascular disease and cancer. Am J Med, 113, 71S-88S.

Ling, W. H.; Cheng, Q. X.; Ma, J. and Wang, T. (2001), Red and black rice decrease atheroscletoric plaque formation and increase antioxidant status in rabbits. $J$ Nutr, 131, 1421-1426.

Manach, C.; Williamson, G.; Morand, C.; Scalbert, A. and Rémesy, C. (2005), Bioavailability and bioefficacy of polyphenols in humans. I. Review of 97 bioavailability studies. Am J Clin Nutr, 81, 230S242S.

Meléndez-Martínez, A. J.; Vicario, I. M. and Heredia, F. J. (2004), Importancia nutricional de los pigmentos carotenoides. Arch Latinoam Nutr, 54, 149-154.

Moran, J. F.; Klucas, R. V.; Grayer, R. J.; Abian, J. and Becana, M. (1997), Complexes of iron with phenolic compounds from soybean nodules and other legume tissues: prooxidant and antioxidant properties. Free Radical Bio Med, 22, 861-870.

Morimitsu, Y.; Kubota, K.; Tashiro, T.; Hashizume, E.; Kamiya, T. and Osawa, T. (2002), Inhibitory effect of anthocyanins and colored rice on diabetic cataract formation in the rat lenses. Int Congr Ser, 1245, 503508.

Nam, S. H.; Choi, S. P.; Kang, M. Y.; Kozukue, N. and Friedman, M. (2005), Antioxidative, antimutagenic, and anticarcinogenic activities of rice bran extracts in chemical and cell assays. J Agric Food Chem, 53, 816-822.

Oki, T.; Masuda, M.; Kobayashi, M.; Nishiba, Y.; Furuta, S.; Suda, I. and Sato, T. (2002), Polymeric procyanidins as radical-scavenging components in red-hulled rice. J Agric Food Chem, 50, 7524-7529.

Parr, A. J. and Bolwell, G. P. (2000), Phenols in the plant and in man. The potential for possible nutritional enhancement of the diet by modifying the phenols content or profile. J Sci Food Agr, 80, 9851012.

Pulido, R.; Bravo, L. and Saura-Calixto, F (2000), Antioxidant activity of dietary polyphenols as determined by a modified ferric reducing/antioxidant power assay. J Agric Food Chem, 48, 3396-3402.
Rossi, J. A. and Kasum, C. M. (2002), Dietary flavonoids: bioavailability, metabolic effects, and safety. Annu Rev Nutr, 22, 19-34.

Santos-Buelga, C. and Scalbert, A. (2000), Proanthocyanidins and tannin-like compounds nature, occurrence, dietary intake and effects on nutrition and health. J Sci Food Agr, 80, 1094-1117.

Simões, C. M. O.; Schenkel, E. P.; Gosmann, G.; Mello, J. C. P.; Mentz, L. A. and Petrovick, P. R. (editors) (2001), Farmacognosia: da planta ao medicamento. Ed. Universidade/UFRGS/Ed. da UFSC, Porto Alegre/Florianópolis.

Stanner, S. A.; Hughes, J.; Kelly, C. N. M and Buttriss, J. (2003), A review of the epidemiological evidence for the 'antioxidant hypothesis'. Public Health Nutr, 7, 407-422.

Tian, S.; Nakamura, K. and Kayahara, H. (2004), Analysis of phenolic compounds in white rice, brown rice, and germinated brown rice. J Agric Food Chem, 52, 4808-4813.

Tian, S.; Nakamura, K.; Cui, T. and Kayahara, H. (2005), High-performance liquid chromatographic determination of phenolic compounds in rice. $J$ Chromatogr A, 1063, 121-128.

Wang, Q.; Han, P.; Zhang, M.; Xia, M.; Zhu, H.; Ma, J.; Hou, M.; Tang, Z. and Ling, W. (2007), Supplementation of black rice pigment fraction improves antioxidant and anti-inflammatory status in patients with coronary heart disease. Asia Pac J Clin Nutr, 16, 295-301.

Xia, M.; Ling, W. H.; Ma, J. and Kitts, D. D. (2003), Supplementation of diets with the black rice pigment fraction attenuates atherosclerotic plaque formation in apolipoprotein E deficient mice. J Nutr, 133, 744751.

Yawadio, R.; Tanimori, S. and Morita, N. (2007), Identification of phenolic compounds isolated from pigmented rices and their aldose reductase inhibitory activities. Food Chem, 101, 1616-1625.

Zhang, M.; Guo, B.; Zhang, R.; Chi, J.; We, Z.; Xu, Z.; Zhang, Y. and Tang, X. (2006), Separation, purification and identification of antioxidant compositions in black rice. Agric Sci China, 5, 431440.

Zhao, Z. and Moghadasian, M. H. (2008), Chemistry, natural sources, dietary intake and pharmacokinetic properties of ferulic acid: a review. Food Chem, 109, 691-702.

Zhou, Z.; Robards, K.; Helliwell, S. and Blanchard, C. (2004), The distribution of phenolic acids in rice. Food Chem, 87, 401-406. 